\title{
D2-40 in breast cancer: should we detect more vascular emboli?
}

\author{
Isabelle de Mascarel ${ }^{1}$, Gaëtan MacGrogan ${ }^{1}$, Marc Debled ${ }^{2}$, Ghislaine Sierankowski ${ }^{1}$, \\ Véronique Brouste ${ }^{3}$, Simone Mathoulin-Pélissier ${ }^{3}$ and Louis Mauriac ${ }^{2}$ \\ ${ }^{1}$ Department of Pathology, Institut Bergonié, Regional Cancer Center, Bordeaux, France; ${ }^{2}$ Department of \\ Medical Oncology, Institut Bergonié, Regional Cancer Center, Bordeaux, France and ${ }^{3}$ Department of \\ Biostatistics, Institut Bergonié, Regional Cancer Center, Bordeaux, France
}

\begin{abstract}
Peritumoral emboli assessed on hematoxylin-eosin-stained slides are taken into account for treatment of patients with operable breast cancer. We assessed whether immunostaining with D2-40 improves the prognostic significance of emboli in a group of tumors with a large immunohistochemical sampling and a longterm follow-up. Topography, number, and extension of hematoxylin-eosin and D2-40 emboli were compared in 94 node-negative breast cancers (median number of immunostained slides per tumor: 3 ). Metastatasis-free survival of patients with or without hematoxylin-eosin and/or D2-40 emboli were evaluated (median follow-up of 178 months). Hematoxylin-eosin emboli were detected in $14(15 \%)$ tumors and were located at distance from the tumor. D2-40 emboli were detected in $39(41 \%)$ tumors and was often multiple $(n=30)$, extensive $(n=23)$, located within $(n=13)$, close to $(n=10)$ or at distance from the tumor $(n=16)$. The 12 distant hematoxylin-eosin and D2-40 emboli were located in the same vessels (seven missed at the first hematoxylin-eosin examination and secondarily diagnosed by D2-40 staining). A difference in metastasis-free survival was found only between patients with no D2-40 emboli and those with distant D2-40 emboli $(P=0.02)$. D2-40 emboli located within or close to the tumor had no prognostic value. Comparing the metastasis-free survival of patients with or without hematoxylin-eosin emboli, the prognostically unfavorable significance of hematoxylin-eosin emboli was improved when taking into account the seven patients with missed emboli at the first examination and secondarily diagnosed by $\mathrm{D} 2-40$ staining $(P=0.006$ vs 0.003$)$. To conclude, D2-40 increases the diagnostic sensitivity of emboli in breast carcinoma and the high incidence of D2-40 emboli might be related to the number of immunostained slides per case. Nevertheless, only distant D2-40 + emboli had a prognostic impact. In practice, D2-40 might be useful to detect missed hematoxylin-eosin emboli especially in cases without any other prognostically unfavorable criterion.
\end{abstract}

Modern Pathology (2009) 22, 216-222; doi:10.1038/modpathol.2008.151; published online 26 September 2008

Keywords: D2-40; lymphovascular invasion; breast cancer; prognosis; immunohistochemistry

The first long-term follow-up studies of node-negative breast cancer that analyzed the prognostic significance of lymphovascular invasion were published in 1977 by Rosen and co-workers ${ }^{1-3}$ Lymphovascular invasion in primary operable breast carcinoma is an essential independent prognostic factor of distant metastasis-free and overall survivals and is taken into account in decisions on adjuvant treatment. ${ }^{4-7}$ In routine practice, the identification of lymphovascular invasion is subject to interobserver variability. Nevertheless, definite peritumoral lymphovascular inva-

Correspondence: Dr I de Mascarel, MD, Department of Pathology, Institut Bergonié, Regional Cancer Center, 229 cours de l'Argonne, Bordeaux 33076, France.

E-mail: mascarel@bergonie.org

Received 25 April 2008; revised and accepted 2 July 2008; published online 26 September 2008 sion in the breast surrounding invasive carcinoma, without distinguishing blood vessels and lymphatics, was found with the same incidence by two teams in $19 \%$ of 1320 and 2760 node-negative patients, respectively., Kahn et al observed that D2-40, a new monoclonal antibody to an $\mathrm{Mr} 40000 \mathrm{O}$-linked sialoglycoprotein, was a sensitive and specific marker of lymphatic endothelium. ${ }^{8,9} \mathrm{D} 2-40$ has mainly been applied as a marker for diagnosing a subset of vascular lesions including Kaposi's sarcoma. ${ }^{10,11}$ D2-40 has also been applied for increasing the detection accuracy of lymphatic invasion in breast carcinomas and melanoma. ${ }^{12,13}$ Moreover, ArnaoutAlkarian et $a l^{12}$ found that lymphatic invasion identified by D2-40 in patients with node-negative breast carcinomas was prognostically significant. The aim of our study was to compare the diagnostic and prognostic value of lymphovascular invasion detected 
on tumor specimens by hematoxylin-eosin and D2-40 in a group of node-negative breast carcinomas.

\section{Materials and methods}

\section{Patients}

Between 1989 and 1992, 937 patients with primary operable invasive breast carcinoma were operated and monitored at our institution. All these patients had been prospectively included by senior pathologists (IM, GMG, IS, JMC) in our clinical, biological and histological database (65 pathological items for noncancerous lesions and 181 for cancers), and a prognostic tissue microarray block was built (GMG). ${ }^{14}$ A small subset of 100 lymph node-negative patients was randomly selected, corresponding to approximately $10 \%$ of the 937 patients. Ninety-four patients were included in this study (blocks not available in six cases). All the patients were treated surgically by either modified radical mastectomy (68 cases) or local tumor resection (26 cases) with axillary node dissection. Postoperative breast irradiation was performed in 69 cases. Adjuvant therapy with chemotherapy and/or hormone therapy was decided according to nodal status and hormone receptor determination results. ${ }^{15,16}$ All patients were followed-up every 3 months for 2 years, twice a year for the next year and then once a year. The median follow-up was 178 months (CI 95\%: 174-183). During this period 13 patients presented distant metastases with or without locoregional recurrences.

\section{Tissue Samples and Histological Examination}

After fixation in Holland-Bouin, the tumor was macroscopically sectioned into three slices. Each slice was put in as many separate cassettes as necessary and paraffin embedded. The median of blocks per patients was $3(1-17)$. In total, 3 blocks per tumor were obtained in 68 cases $(72 \%)$ and $4-17$ blocks in 14 cases. Each block was examined on one hematoxylin-eosin section/slide. Original hematoxylin-eosin slides of the tumor were not reviewed. The diameter of the tumor (histological size) was measured on fresh specimens and on hematoxylin-eosin slides. The largest diameter was taken into account. A modified SBR-grading system grade was used. ${ }^{17}$

\section{Immunohistochemical Assay}

For the study, each tumor block from the 94 patients was recut into $4 \mu \mathrm{m}$ sections mounted on SuperFrost ${ }^{\circledR}$ slides, and one slide per block was stained with D2-40. Thus, 324 D2-40-stained slides were analyzed. Antigen retrieval was obtained by heating tissue sections, immersed in a $0.01 \mathrm{M}$ citrate buffer, in a pH9 solution (Dako ${ }^{\circledR}$ S2367), in a pressure cooker for $20 \mathrm{~min}$. The sections were then incubated for $60 \mathrm{~min}$ with D2-40 (Dako ${ }^{\mathbb{R}}$, dilution 1/50). A labeled streptavidin-biotin-peroxidase method (LSAB kit K5001, DakoCytomation ${ }^{\circledR}$, France) was used to visualize the immunoreactions with diaminobenzidine as a chromogen. Immunohistochemical stainings were analyzed by one pathologist (IM) blind to whether the case was hematoxylin-eosin positive or negative for lymphovascular invasion. As underlined by Kahn et al ${ }^{10,11}$ and Fukunaga, D2-40 selectively stained flattened or open lymphatic channels lined by a single layer of endothelial cells, whose lumen was sometimes filled with lymphocytes. D2-40 did not stain blood vessels: capillaries and the endothelium of arteries were negative or rarely weakly positive.
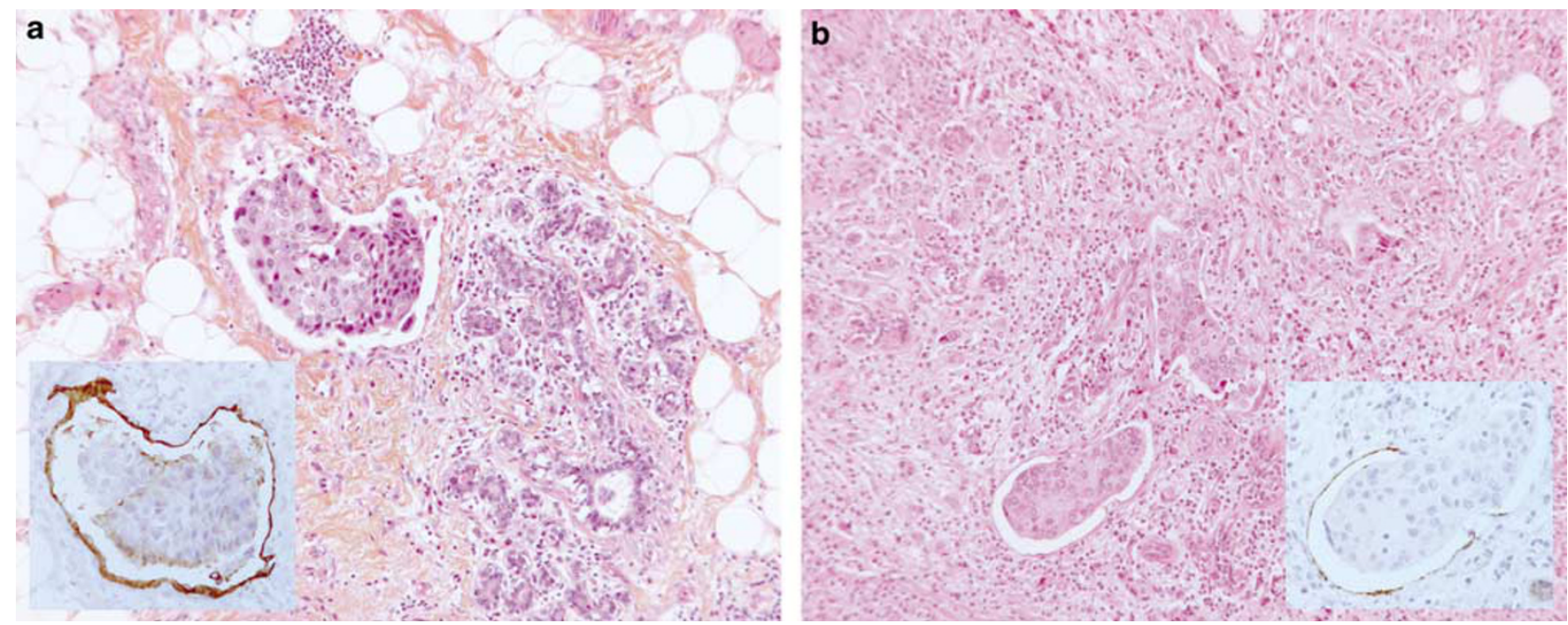

Figure 1 (a) Distant peritumoral vessel with definite tumoral invasion close to a normal lobule; inset: D2-40 immmunostaining. (b) Intratumoral vessel with tumoral invasion; inset: D2-40 immmunostaining. 


\section{Definition of Lymphovascular Invasion}

Definition of lymphovascular invasion detected by hematoxylin-eosin

Definite peritumoral lymphovascular invasion on original hematoxylin-eosin-stained slides was defined as carcinoma cells present within a definite endothelial lined space, at a distance from the tumor (Figure 1a), in the breast surrounding invasive carcinoma, ie separated from the tumor by normal breast tissue and often next to a small artery or vein. ${ }^{4,18}$ When hematoxylin-eosin lymphovascular invasion was suspected in the vicinity of the tumor, differential diagnosis with an artifact (retraction) was difficult, and such equivocal cases were recorded as negative. In our study, only unequivocal hematoxylin-eosin lymphovascular invasion was recorded as positive without distinguishing blood vessels from lymphatics. There were 14 (15\%) patients with definite hematoxylin-eosin distant peritumoral lymphovascular invasion and this is exactly the frequency expected. ${ }^{19}$ Although hematoxylin-eosin emboli were not counted, they were single in most cases. Patient characteristics are summarized in Table 1.

Definition of lymphatic invasion detected by D2-40 Number and topography (intra and/or peritumoral) of lymphovascular invasion detected by D2-40 (D2-40 + LVI) was recorded (Figure 1a and b). Close peritumoral D2-40 + LVI was seen at the invasive front of the tumor, ie close to tumor cells or desmoplastic stroma. The definition of distant $\mathrm{D} 2-40+$ lymphovascular invasion was similar to the one of distant peritumoral hematoxylin-eosin lymphovascular invasion, ie separated from the tumor by normal breast tissue. Only obvious peritumoral emboli in unequivocal vessels detected with D2-40 were recorded as positive. Equivocal cases $(n=10)$ were analyzed by two pathologists (IM and GMG), and recorded as negative when results between the two pathologists ( $n=10$ cases) were discordant. In each case, the number of 'positive' blocks/slides (ie number of slides with definite emboli) was assessed. Extensive distant peritumoral vascular invasion was defined as one or more emboli on more than one tumor block/slide according to Colleoni et $a{ }^{20}$ criteria.

\section{Comparison of Hematoxylin-Eosin and D2-40 Slides}

When lymphovascular invasion was detected simultaneously by hematoxylin-eosin and D2-40, slides were reviewed to assess whether it was the same embolus in the same vessel. Slides of discordant cases were also reviewed to assess precisely, on one hand, the presence or the absence of distant peritumoral vessels on the recuts from the corresponding block, and on the other, the topography of lymphovascular invasion. Hemato-
Table 1 Clinicopathological data of patients included

\begin{tabular}{|c|c|c|}
\hline Patients & $\mathrm{N}$ & $\%$ \\
\hline \multicolumn{3}{|l|}{ Age } \\
\hline$\leq 50$ years & 28 & 30 \\
\hline$>50$ years & 66 & 70 \\
\hline \multicolumn{3}{|l|}{ Histological size } \\
\hline$\leq 20 \mathrm{~mm}$ & 76 & 81 \\
\hline$>20 \mathrm{~mm}$ & 14 & 15 \\
\hline Not specified & 4 & 4 \\
\hline \multicolumn{3}{|l|}{ Bloom-Richardson grade } \\
\hline 1 & 27 & 29 \\
\hline 2 & 42 & 45 \\
\hline 3 & 25 & 26 \\
\hline \multicolumn{3}{|l|}{ Hematoxylin-eosine emboli } \\
\hline Absent & 80 & 85 \\
\hline Present & 14 & 15 \\
\hline \multicolumn{3}{|l|}{ Hormonal receptors ${ }^{\mathrm{a}}$} \\
\hline Estrogen and Progesterone (-) & 19 & 20 \\
\hline Estrogen and Progesterone (+) & 48 & 51 \\
\hline Estrogen $(-)$ and Progesterone (+) & 4 & 4 \\
\hline Estrogen $(+)$ and Progesterone $(-)$ & 16 & 17 \\
\hline Not specified & 7 & 8 \\
\hline \multicolumn{3}{|l|}{$M_{i b 1}{ }^{\mathrm{a}}$} \\
\hline$<20 \%$ & 63 & 67 \\
\hline$\geq 20 \%$ & 24 & 25 \\
\hline Not specified & 7 & 8 \\
\hline \multicolumn{3}{|l|}{ Her2neu ${ }^{\mathrm{a}}$} \\
\hline$<10 \%$ & 74 & 79 \\
\hline$\geq 10 \%$ intensity 2 & 3 & 3 \\
\hline$\geq 10 \%$ intensity 3 & 13 & 14 \\
\hline Not specified & 4 & 4 \\
\hline
\end{tabular}

${ }^{a}$ Assessed on tissue microarray: estrogen receptor and progesterone receptor : $<10 \%$ of positive tumor cells $=$ negative; $\geq 10 \%$ of positive tumor cells = positive. Mib1: $<20 \%$ of positive cells =low; $\geq 20 \%=$ high. Score Herceptest: $10 \%$, intensity $1-3 ; 0 \%$ and $1=$ no overexpression, $\geq 10 \%$ and 2 or $3=$ overexpression.

xylin-eosin lymphovascular invasion that had been missed at first examination and diagnosed at the second in the light of D2-40 findings.

\section{Statistics}

Correlations between vascular invasion and patient characteristics were studied with the $\chi^{2}$ - and Wilcoxon tests. Metastasis-free survival was calculated according to the Kaplan-Meier method from the date of the surgery to the occurrence of distant metastasis. We compared MFS between the patients with and without vascular invasion using the log-rank test (SPSSv14).

\section{Results}

\section{Lymphatic Invasion Detected by D2-40}

D2-40 lymphovascular invasion was present in 39 $(41 \%)$ out of the 94 cases and was located in or at the 
periphery of the tumor in 13 and 26 cases, respectively. Peritumoral D2-40 lymphovascular invasion was close to the tumor in 10 cases and distant in 16 (in association with close D2-40 + LVI in 12 cases). D2-40 lymphovascular invasion was multiple (on the same slide or on different slides) in 30 cases (3-14 emboli in 21 cases). Thus, 10 of 13 intra, 8 of 10 close and 12 of 16 distant and close D240 lymphovascular invasion were multiple. D2-40 lymphovascular invasion was extensive in 23 cases (three slides or more in 11cases). Thus, 7 of 13 intra, 5 of 10 close and 9 of 16 distant and close D2-40 lymphovascular invasion were extensive. Finally, D2-40 lymphovascular invasion was present on only one out of the three or more sections/slides in 16 of $39(41 \%)$ cases.

\section{D2-40 and Patient Characteristics}

D2-40 lymphovascular invasion were more frequent in younger patients (mean age 38 vs 54 years in positive and negative patients, respectively, $P=0.005$ ) and in larger tumors (mean size $53 \mathrm{Vs}$ $40 \mathrm{~mm}$ in positive and negative patients, respectively, $P=0.015)$. No correlation was found between hormonal receptor status and histological tumor grade.

\section{Comparison of Hematoxylin-Eosin and D2-40 in Concordant and Discordant Cases}

Concordant cases: concurrent positivity or negativity of hematoxylin-eosin and D2-40

Hematoxylin-eosin and D2-40 were simultaneously negative in 47 cases and positive in 6 cases (Table 2). In the six tumors with hematoxylin-eosin and D2-40 lymphovascular invasion, D2-40 lymphovascular invasion was often multiple (five cases with five or more lymphovascular invasion) and located in $(n=1)$ or at distance from the tumor $(n=5)$, whereas hematoxylin-eosin lymphovascular invasion was

Table 2 D2-40 emboli according to absence or presence of hematoxylin-eosin emboli

\begin{tabular}{lcc}
\hline & $\begin{array}{c}\text { No hematoxylin-eosin } \\
\text { emboli }(\mathrm{n}=80)\end{array}$ & $\begin{array}{c}\text { Hematoxylin-eosin } \\
\text { emboli }(\mathrm{n}=14)\end{array}$ \\
\hline $\begin{array}{l}\text { No D2-40 } \\
\text { emboli }\end{array}$ & 47 & 8 \\
$\begin{array}{l}\text { D2-40 emboli } \\
\text { Intratumoral }\end{array}$ & 12 & 1 \\
$\begin{array}{l}\text { Peritumoral } \\
\text { Close } \\
\begin{array}{l}\text { Close and } \\
\text { distant }\end{array}\end{array}$ & 10 & - \\
Distant & 7 & 5 \\
\hline
\end{tabular}

${ }^{\mathrm{a}}$ Associated with intratumoral emboli in one case. single and located in distant vessels. The five single distant peritumoral emboli detected both by hematoxylin-eosin and D2-40 were located in the same vessels.

Discordant cases: positivity of hematoxylin-eosin or D2-40

There were 41 cases with one positive staining: 8 cases with lymphovascular invasion detected by hematoxylin-eosin only and 33 with lymphovascular invasion detected by D2-40 only (12 intra, 10 close and 11 distant LVI). When comparing the eight hematoxylin-eosin and D2-40-stained slides on which lymphovascular invasion had been detected by hematoxylin-eosin only, distant vessels with hematoxylin-eosin lymphovascular invasion were actually absent on the D2-40-stained slides corresponding to recuts from the corresponding block. When comparing the 11 hematoxylin-eosin and D2-40-stained slides on which distant lymphovascular invasion had been detected by D2-40 only, 7 hematoxylin-eosin lymphovascular invasion had been missed at the first hematoxylin-eosin examination and diagnosed at the second by D2-40 in the same distant lymphatic vessels, and 4 D2-40 lymphovascular invasion were located in vessels that were absent on the original hematoxylin-eosinstained slides. Finally, lymphovascular invasion detected both by hematoxylin-eosin and D2-40 was located in the same distant vessels in 12 cases ( 5 cases at the first examination and 7 at the second). Among the seven hematoxylin-eosin lymphovascular invasion missed at the first examination, only one was found in a SBR grade 3 tumor. Using hematoxylin-eosin lymphovascular invasion as gold standard, D2-40 sensitivity and specificity were respectively $42.8 \%$ (95\% CI: 17.6-71.1) and 58.7\% (95\% CI: 47.1-69.6).

\section{Prognostic Significance of Lymphovascular Invasion According to Staining Used}

There was no significant difference in metastasisfree survival between patients with or without D2-40 lymphovascular invasion $(P=0.14)$ whatever their extension $(P=0.2)$. When taking into account the topography of D2-40 lymphovascular invasion, a difference in survival was found only between patients with no D2-40 lymphovascular invasion and those with distant D2-40 lymphovascular invasion $(P=0.02$, Figure 2a). D2-40 lymphovascular invasion located within or close to the tumor had no prognostic value. Moreover, a significant difference was also found between patients with fewer or more than two D2-40 emboli $(P=0.04)$. Among the 13 tumors that subsequently relapsed, hematoxylin-eosin lymphovascular invasion was diagnosed in 5 cases at first examination and in 2 cases secondarily by D2-40 staining (no relapse in the 4 tumors with D2-40+/negative lymphovascular 

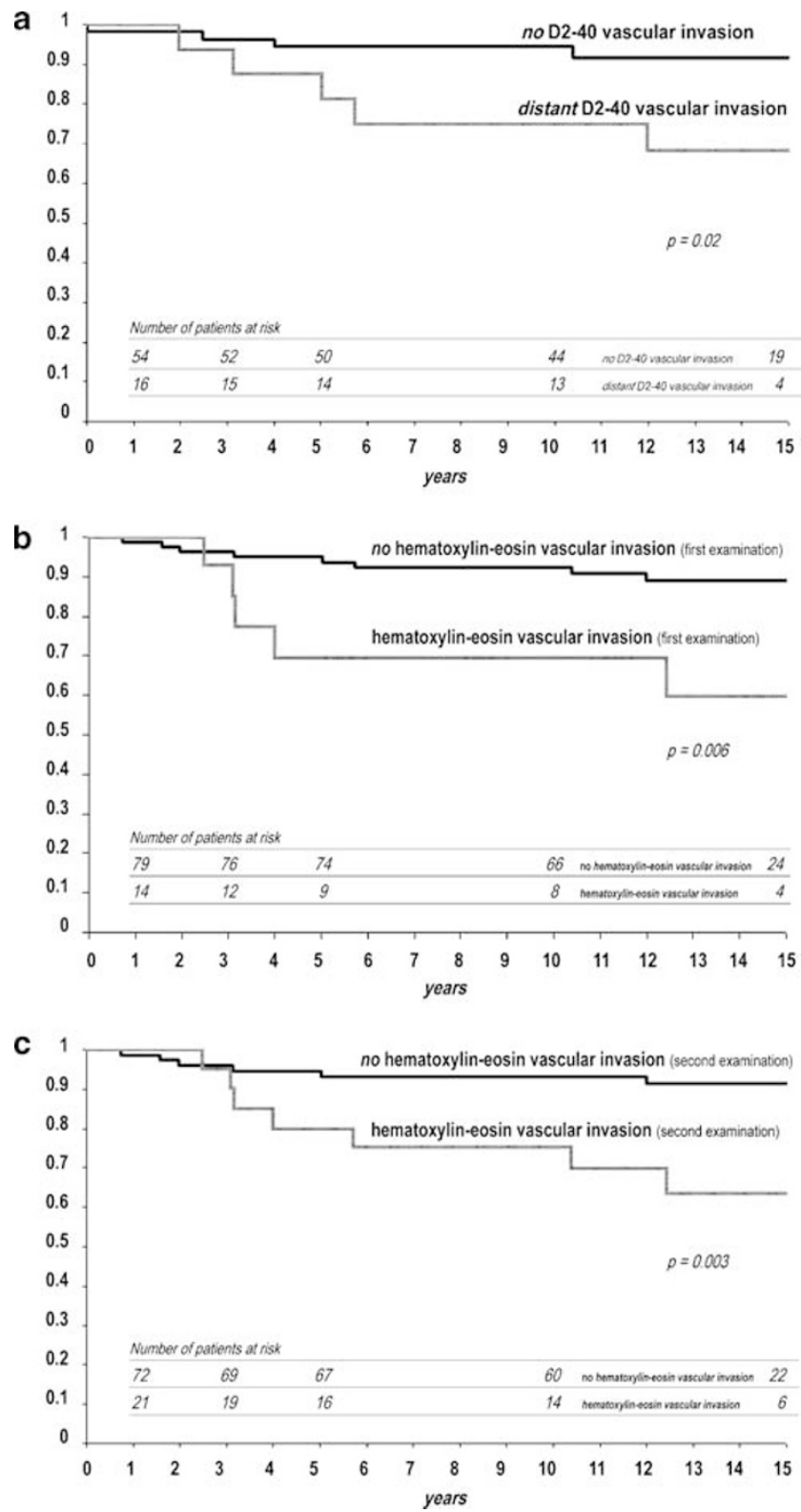

Figure 2 Metastasis-free survival curves according to the absence or presence of lymphovascular invasion detected by hematoxylin-eosin and D2-40. (a) Metastasis-free survival according to presence or absence of distant lymphovascular invasion detected by D2-40. (b) Metastasis-free survival according to presence or absence of distant lymphovascular invasion detected by hematoxylin-eosin at the first examination. (c) Metastasis-free survival according to presence or absence of distant lymphovascular invasion detected by hematoxylin-eosin at the second examination in the light of D2-40 findings.

invasion at review). Comparing the metastasis-free survival of patients with or without hematoxylineosin lymphovascular invasion, the prognostically unfavorable significance of hematoxylin-eosin lymphovascular invasion was improved when taking into account D2-40 lymphovascular invasion confirmed by hematoxylin-eosin $(P=0.006$ vs 0.003 , Figure $2 \mathrm{~b}$ and $\mathrm{c}$ ).

\section{Discussion}

Incidence of Lymphatic Invasion Detected by D2-40

The lower incidence of D2-40 lymphovascular invasion in the others studies: $27,{ }^{12} 23,{ }^{21} 15 \%{ }^{22}$ compared to the incidences in our study (41\%) and in that by Van den Eynden et $a l^{23}(70 \%)$ may be due to the number of immunostained sections/ slides per tumor: one ${ }^{12,21,22}$ vs four $^{23}$ and a mean of 3 in the present study. Furthermore, Van den Eynden et $a l^{23}$ included node-positive patients with large tumors.

\section{Discordances Between Hematoxylin-Eosin and D2-40}

Positivity of hematoxylin-eosin and negativity of D2-40

Four hypotheses can be made: (1) vascular lumina are absent on immunostained slides corresponding to recuts from the corresponding block (eight cases in our study); (2) vascular lumina are present but without emboli; (3) hematoxylin-eosin lymphovascular invasion corresponds to artifacts; (4) hematoxylin-eosin emboli are located in vascular channels that are not lymphatics. Nevertheless, Mohammed et $a l^{21}$ recently found that blood vascular invasion was rare comparatively to lymphovascular invasion (3.5 vs $96.4 \%)$.

\section{Negativity of hematoxylin-eosin and positivity of D2-40}

These cases were more numerous in our series than in that of Arnaout-Alkarian et al $(41 \%$ vs $18 \%$, respectively). ${ }^{12}$ In this situation, three hypotheses can be made: (1) emboli are missed at the first hematoxylin-eosin examination and subsequently scored in the light of D2-40 findings (seven cases in our study); (2) emboli are present at the first hematoxylin-eosin but cannot be taken into account: they are not located at distance from the tumor (23 intra or close lymphovascular in our study) and/or the complete obliteration of vascular lumina does not allow a diagnosis of lymphovascular invasion; (3) vascular lumina are absent on hematoxylin-eosin slides and present on immunostained slides corresponding to recuts from the corresponding block (four cases in our study).

Thus, the 12 cases with lymphatic vessels absent either on hematoxylin-eosin or on D2-40-stained slides cannot be considered with certainty as 'true' discordant cases.

\section{Prognostic Significance of D2-40 Lymphovascular Invasion According to Topography, Number, and Extension}

Although the median follow-up was long in our study, the relatively small number of patients and events requires caution when interpreting these 
results. With a comparable median follow-up (8 years), some authors ${ }^{12,21}$ found that D2-40 lymphovascular invasion (topography not specified) was a significant predictor of outcome. Furthermore, when comparing the pejorative significance of hematoxylin-eosin and D2-40 lymphovascular invasion, the pejorative significance of D2-40 lymphovascular invasion was stronger $(P=0.05$ vs $P=0.007) .{ }^{21}$ In our study, the clinical impact of distant D2-40 lymphovascular only and the stronger prognostic impact of hematoxylin-eosin lymphovascular invasion when taking into account missed hematoxylin-eosin lymphovascular invasion raises the hypothesis that D2-40 could detect different types of lymphatic vessels or emboli. On one hand, there could be emboli located in normal preexistent mature lymphovascular channels found in the breast surrounding invasive carcinoma; and on the other, emboli located in lymphatic neocapillaries within the tumor with a chaotic architecture and abnormal function. Vascular proliferation is now known to be heterogeneous in tumoral neoangiogenesis and vasculogenesis. Lymphatic emboli located in 'abnormal' lymphatic neovessels within or close to the tumor could be different from lymphatic emboli located in normal lymphatic capillaries and mature lymphatic vascular channels located outside the tumor, ie connection between the two systems may not be efficient. Furthermore, various studies have suggested that the growth of tumor-associated lymphatic vessels induced by vascular endothelial growth factors $\mathrm{C}$ and $\mathrm{D}$ promotes lymphatic invasion. ${ }^{24-28}$ Increased lymphovascular tumor density was found to be a significant unfavorable prognostic factor in breast cancer, ${ }^{28,29}$ melanoma $^{30,31}$ and colorectal cancer. $^{32}$ Some authors ${ }^{33}$ found in 89 cases of breast cancer that lymphangiogenesis was mainly located in tumor stroma, whereas mature lymphatic vessels were not found in cancer nests. Such a distinction between intra- and peritumoral lymphatic vessels was also underlined by Massi et $a l^{30}$ in 45 cases of melanoma. In their study, peritumoral lymphovascular density was an independent variable affecting overall survival, whereas intratumoral lymphovascular density was the most significant predictor of sentinel lymph node metastasis. Additional studies are necessary to analyze more precisely the relationship between intra- and peritumoral lymphangiogenesis, intraand peritumoral lymphatic invasion and clinical outcome (lymph node metastases, distant metastasis and overall survival).

\section{Practical Considerations}

Definitions of hematoxylin-eosin lymphovascular invasion

As hematoxylin-eosin lymphovascular invasion had been missed in 7 cases out of $22(33 \%)$ at the first hematoxylin-eosin examination, the term 'obvious' peritumoral emboli as defined in a previous work is no longer adequate. ${ }^{4}$ On the contrary, the term 'unequivocal lymphatic vessels' is still adequate as hematoxylin-eosin emboli have to be located in unequivocal distant D2-40-positive lymphatic vessels.

Is it useful to detect more lymphatic tumor emboli by D2-40 in routine practice?

The stronger prognostic impact of hematoxylineosin lymphovascular invasion when adding the missed lymphovascular invasion detected by D2-40 suggests an affirmative answer to this question. Nevertheless, it is not necessary to search for all emboli in all patients. D2-40 could be used in nodenegative patients without hematoxylin-eosin emboli and without any other prognostically unfavorable pathologic criterion (ie with a tumor SBR grade 1 or 2 sizing less than $2 \mathrm{~mm}$, with positive hormonal receptors and negative Her2Neu status) to search for missed distant hematoxylin-eosin lymphovascular invasion on all tumor blocks. Thus, in our study group, D2-40 would have been performed in nearly half of patients $(n=48)$ and would allow the detection of 6 missed distant hematoxylin-eosin lymphovascular invasion (12.5\%, CI 95\%: $4.7-$ $25 \%)$.

In conclusion, our study raises several hypotheses: (1) the high incidence of D2-40 lymphovascular invasion might be related to the number of immunostained slides per case; (2) D2-40 might detect different types of vessels/lymphovascular invasion; and (3) only distant D2-40 lymphovascular invasion confirmed by hematoxylin-eosin might have a prognostic impact. Thus, in routine practice, D2-40 might be useful especially for novice pathologists to detect missed hematoxylin-eosin emboli. Further studies with large cohorts of patients are necessary to assess accurately the clinical significance of D2-40 lymphatic invasion. This is a time-consuming and expensive undertaking as it cannot be performed on tissue microarray blocks. However, it is of practical interest as this reliable, reproducible and routinely applicable factor is of prognostic value.

\section{Acknowledgements}

We thank Laure Gluais for her technical assistance. This study was funded by a $100 \%$ grant of L Mauriac to the Department of Pathology in June 2005.

\section{References}

1 Nime F, Rosen PP, Thaler $\mathrm{H}$, et al. Prognostic significance of tumor emboli in intramammary lymphatics in patients with mammary carcinoma. Am J Surg Pathol 1977;1:25-30. 
2 Rosen PP, Saigo PE, Braun DW, et al. Predictors of recurrence in stage I (T1N0M0) breast carcinoma. Ann Surg 1981;193:15-25.

3 Rosen PP. Tumor emboli in intramammary lymphatics in breast carcinoma: pathologic criteria for diagnosis and clinical significance. Pathol Annu 1983;18(Part 2): 215-232.

4 de Mascarel I, Bonichon F, Durand M, et al. Obvious peritumoral emboli: an elusive prognostic factor reappraised. Multivariate analysis of 1320 node-negative breast cancers. Eur J Cancer 1998;34:58-65.

5 Lee AH, Pinder SE, Macmillan RD, et al. Prognostic value of lymphovascular invasion in women with lymph node negative invasive breast carcinoma. Eur J Cancer 2006;42:357-362.

6 Trudeau ME, Pritchard KI, Chapman JA, et al. Prognostic factors affecting the natural history of nodenegative breast cancer. Breast Cancer Res Treat 2005;89:35-45.

7 Goldhirsch A, Coates AS, Gelber RD, et al. First-select the target: better choice of adjuvant treatments for breast cancer patients. Ann Oncol 2006;17: 1772-1776.

8 Kahn HJ, Marks A. A new monoclonal antibody, D2-40, for detection of lymphatic invasion in primary tumors. Lab Invest 2002;82:1255-1257.

9 Marks A, Sutherland DR, Bailey D, et al. Characterization and distribution of an oncofetal antigen (M2A antigen) expressed on testicular germ cell tumors. Br J Cancer 1999;80:569-578.

10 Kahn HJ, Bailey D, Marks A. Monoclonal antibody D240, a new marker of lymphatic endothelium, reacts with Kaposi's sarcoma and a subset of angiosarcomas. Mod Pathol 2002;15:434-440.

11 Fukunaga M. Expression of D2-40 in lymphatic endothelium of normal tissues and in vascular tumours. Histopathol 2005;46:396-402.

12 Arnaout-Alkarain A, Kahn HJ, Narod SA, et al. Significance of lymph vessel invasion identified by the lymphatic marker D2-40 in node negative breast cancer. Mod Pathol 2007;20:183-191.

13 Niakosari F, Kahn HJ, Marks AN, et al. Detection of lymphatic invasion in primary melanoma with monoclonal antibody D2-40: a new selective immunohistochemical marker of lymphatic endothelium. Arch Dermatol 2005;141:440-444.

14 Bui Nguyen Binh-Desrousseaux M. Mise en place d'un tissu micro-array pronostique dans le cancer du sein. A propos de 855 cas de carcinomes canalaires infiltrants. Université de Bordeaux 2 2005, Thèse $\mathrm{N}^{\circ} 3058$ (submitted).

15 Mauriac L, Durand M, Chauvergne J, et al. Adjuvant trial for stage II receptor positive breast cancer: CMF vs $\mathrm{CMF}+$ tamoxifen in single centre. Breast Cancer Res Treat 1988;11:179-186.

16 Mauriac L, Durand M, Chauvergne J, et al. Cancers of the breast at metastatic high-risk: prediction of survival by steroidal receptors. Les cancers du sein à haut risque métastatique: prédiction de la survie par les récepteurs stéroidiens. Bull Cancer Paris 1989;76:33-41.

17 Elston CW, Ellis IO. Pathological prognostic factors in breast cancer. I. The value of histological grade in breast cancer: experience from a large study with long-term follow-up. Histopathology 1991;19:403-410.
18 Rosen PP, Oberman HA. Tumors of the mammary gland. Washington: Armed Forces Institute of Pathology, 1993, $390 \mathrm{p}$.

19 Rosen PP. Rosen's Breast Pathology, 2nd edn. Lippincot Williams \& Wilkins editor: Philadelphia, 2001.

20 Colleoni M, Rotmensz N, Maisonneuve $\mathrm{P}$, et al. Prognostic role of the extent of peritumoral vascular invasion in operable breast cancer. Ann Oncol 2007;18:1632-1640.

21 Mohammed RA, Martin SG, Gill MS, et al. Improved methods of detection of lymphovascular invasion demonstrate that it is the predominant method of vascular invasion in breast cancer and has important clinical consequences. Am J Surg Pathol 2007;31:1825-1833.

22 Braun M, Flicke U, Debald M, et al. Detection of lymphovascular invasion in early breast cancer by D2-40 (podoplanin): a clinically useful predictor for axillary lymph node metastases. Breast Cancer Res Treat 2007; e-pub ahead of print.

23 Van den Eynden GG, Van der Auwera I, Van Laere SJ, et al. Distinguishing blood and lymph vessel invasion in breast cancer. A prospective immunohistochemical study. Br J Cancer 2006;94:1643-1649.

24 Karpanen T, Egeblard M, Karkkainen MJ, et al. Vascular endothelial growth factor C promotes tumout lymphangiogenesis and intralymphatic tumor growth. Cancer Res 2001;61:1786-1790.

25 Mandriota SJ, Jussila L, Jeltsch M, et al. Vascular endothelial growth facto-C-mediated lymphangiogenesis promotes tumour metastasis. EMBO J 2001;20:672-682.

26 Stacker SA, Stacker SA, Caesar C, et al. VEGF-D promotes the metastatic spread of tumor cells via the lymphatic. Nat Med 2001;7:186-191.

27 Skobe M, Hawighorst T, Jackson DG, et al. Induction of tumor lymphangiogenesis by VEGF-C promotes breast cancer metastasis. Nat Med 2001;7:192-198.

28 Schoppmann SF, Fenzl A, Nagy K, et al. VEGF-C expressing tumor-associated macrophages in lymph node positive breast cancer: impact on lymphangiogenesis and survival. Surgery 2006;139: 839-846.

29 Nakamura Y, Yasuoka H, Tsujimoto $\mathrm{M}$, et al. Lymph vessel density correlates with nodal status, VEGF-C expression, and prognosis in breast cancer. Breast Cancer Res Treat 2005;91:125-132.

30 Massi D, Puig S, Franchi A, et al. Tumor lymphangiogenesis is a possible predictor of sentinel lymph node status in cutaneous melanoma: a case-control study. J Clin Pathol 2006;59:166-173.

31 Dadras SS, Lange-Asschenfeldt B, Velasco $\mathrm{P}$, et al. Tumor lymphangiogenesis predicts melanoma metastasis to sentinel lymph nodes. Mod Pathol 2005;18:1232-1242.

32 Jia YT, Li ZX, He YT, et al. Expression of vascular endothelial growth factor-C and the relationship between lymphangiogenesis and lymphatic metastasis in colorectal cancer. World J Gastroenterol 2004;10:3261-3263.

33 Huang JH, Li Y, Liu L, et al. Lymphangiogenesis and location of tumor lymphatic vessels induced by VEGF$\mathrm{C}$ in primary breast cancer. Zhong Nan Da Xue Xue Bao Yi Xue Ban 2006;31:36-39, 51. 